\title{
Augmenting a Conceptual Model with Geospatiotemporal Annotations
}

\author{
Vijay Khatri, Member, IEEE, Sudha Ram, Member, IEEE, and \\ Richard T. Snodgrass, Senior Member, IEEE
}

\begin{abstract}
While many real-world applications need to organize data based on space (e.g., geology, geomarketing, environmental modeling) and/or time (e.g., accounting, inventory management, personnel management), existing conventional conceptual models do not provide a straightforward mechanism to explicitly capture the associated spatial and temporal semantics. As a result, it is left to database designers to discover, design, and implement-on an ad hoc basis-the temporal and spatial concepts that they need. We propose an annotation-based approach that allows a database designer to focus first on nontemporal and nongeospatial aspects (i.e., "what") of the application and, subsequently, augment the conceptual schema with geospatiotemporal annotations (i.e., "when" and "where"). Via annotations, we enable a supplementary level of abstraction that succinctly encapsulates the geospatiotemporal data semantics and naturally extends the semantics of a conventional conceptual model. An overarching assumption in conceptual modeling has always been that expressiveness and formality need to be balanced with simplicity. We posit that our formally defined annotation-based approach is not only expressive, but also straightforward to understand and implement.
\end{abstract}

Index Terms-Data semantics, database design, semantic model, geospatial databases, temporal databases.

\section{INTRODUCTION}

$\mathrm{M}$ ANY real-world georeferenced (e.g., land information systems, environmental modeling, transportation planning, geomarketing, geology, archaeology) and timevarying (e.g., accounting, portfolio management, personnel management, inventory management) applications need to organize data based on space and/or time. Underlying these applications are temporal and/or geospatial data, collectively referred to as geospatiotemporal data. Conceptual database design is widely recognized as an important step in the development of database applications [1], [3], [7] such as those listed above. During conceptual database design, a conceptual model provides a notation and formalism that can be used to construct a high-level description of the real world-referred to as a conceptual schema-independent of implementation details. The data semantics provides a mapping from the conceptual schema to aspects in the real world. However, conventional conceptual models [1], [3], [7] do not provide a straightforward mechanism to explicitly capture the semantics related to space and time. As a result, it is left to the database designers to discover, design and implement-on an ad hoc basis-the temporal and spatial concepts that they need. In this paper, we present a methodical approach that augments a conventional conceptual model using geospatiotemporal annotations.

- V. Khatri is with the Information Systems Department, Kelley School of Business, Indiana University, 1309 East 10th Street, BU 572, Bloomington, IN 47405-1701. E-mail: vkhatri@indiana.edu.

- S. Ram is with the Department of MIS, University of Arizona, 1130 E. Helen St., Tucson, AZ 85721-0108. E-mail: ram@eller.arizona.edu.

- R.T. Snodgrass is with the Department of Computer Science, University of Arizona, 711 Gould Simpson, PO Box 210077, Tucson, AZ 85721-0077. E-mail: rts@cs.arizona.edu.

Manuscript received 10 Jan. 2002; revised 26 Aug. 2003; accepted 13 Oct. 2003.

For information on obtaining reprints of this article, please send e-mail to: tkde@computer.org, and reference IEEECS Log Number 115685.
Many prior studies [10], [23] attribute project failures to lack of identifying real needs during conceptual design. One of the problems with developing geospatiotemporal applications is that there is "a gulf between the richness of knowledge structures in the application domains and the relative simplicity of the data model in which the structures can be expressed" [33], which in turn impacts the ability to elicit the application requirements. Considering that geographic data are finding their way into traditional applications (e.g., insurance, retail, distribution), there is a need for an overall geospatiotemporal conceptual database design methodology that can be integrated into conventional conceptual design. Thus, it would be helpful to develop an approach that is compatible with an existing generalpurpose methodology [1], [3], [7].

Our annotation-based approach divides geospatiotemporal conceptual design into two steps: 1) elicit the current reality of an application using a conventional conceptual model without considering the geospatial and temporal aspects ("what") and, only then, 2) annotate the schema with the geospatiotemporal semantics of the application ("when" and "where"). Rather than creating new constructs in a conceptual model, we use annotations to elicit the geospatiotemporal aspects of the application. Our annotationbased approach is generic and can be applied to any conventional conceptual model [1], [3], [7] to transform that model into a geospatiotemporal conceptual model. In this paper, we apply our annotation-based approach to the Unifying Semantic Model (USM) [22]—an extended version of the Entity-Relationship (ER) Model [3]—-to propose the geoSpatioTemporal Unifying Semantic Model (ST USM).

We mention here the assumptions in this paper to delineate the scope of our work.

1. Based on perception, space may be differentiated as large-scale and small-scale [17]. As with Mark and Frank [19], we construe large-scale space as equivalent to geographic space. In the following, we use the 
term space interchangeably to mean large-scale space or geographic space.

2. According to Peuquet [21], absolute space is objective since it provides an immutable structure that is purely geometric. On the other hand, relative space is an ordering relation between objects that determines their relative position. We concentrate on absolute representations, which are typically employed in databases.

3. A database schema can evolve with time. Schema versioning [24] is an important area of research; however, we do not focus on schema versioning.

In summary, this paper focuses on establishing a foundation for capturing the geospatiotemporal data semantics during conceptual design and does not delve into peripheral research areas.

The rest of the paper is organized as follows: In Section 2, we outline requirements related to geospatiotemporal conceptual modeling. According to Wand et al. [31], "the power of a modeling language lies in the semantics of its constructs" and "ontology can be used to define concepts that should be represented by the modeling language." The basis for annotations is the time and space ontology summarized in Section 3. We describe our annotationbased geospatiotemporal conceptual design methodology, which first focuses on "what" is important for an application in the real world and then associates "what" with "when" and/or "where." In Section 4, we summarize a conventional conceptual model, USM, which provides various abstractions to capture "what" is important for an application. In Section 5, we apply our annotation-based approach to USM to realize ST USM, which captures the semantics related to "when" and/or "where." We round out the paper with an evaluation and contributions. In this paper, we provide the essence of our approach; complete details are available in a comprehensive report [15].

\section{Desiderata}

A precursor to designing and developing a geospatiotemporal conceptual model is identifying the conceptual modeling requirements that need to be met. Based on a hydrogeologic study at the US Geological Survey (USGS), we provide an example of an application that needs to capture the geospatiotemporal data semantics. We then outline the evaluation criteria for a conceptual model that can capture the data semantics for geospatiotemporal applications like that at the USGS.

\subsection{Hydrogeologic Application}

We are working with a group of researchers who are developing a ground-water flow model [5] for the Death Valley region in the state of Nevada. Beneath the earth's surface, there is a zone where all interstices are filled with water referred to as ground water. The objective of the ground-water flow model is to characterize regional 3D ground-water flow paths so that policy-makers can make decisions related to radionuclide contaminant transport and the impact of ground water pumping on national parks and local communities in the region. However, the quality of the model output and the predictions based on these models are dependent on the data that forms an input to these models.

A large part of the input data for this model is geospatial and/or temporal in nature. For example, two key objects of interest in the application are spring-water sites and borehole sites. Both these objects need to be spatially referenced to the earth. A spring-water site is represented as a point whose location on the surface of the earth is given by the geographic $x$ and $y$-coordinates, with a geospatial granularity of degree. Spring-water sites are points where spring discharge is measured. Similar to springs, boreholes are access points to the ground water system. Information of their construction and condition are important for monitoring ground water supply and remediation. A borehole site refers to a part of the borehole whose 3D location is given by the $x$ and $y$-coordinates on the earth's surface with a geospatial granularity of degree, along with the depth below land surface with a geospatial granularity of foot; there can be different borehole sites at different depths at the same surface location. Physically, a borehole is composed of hole-intervals with different diameters. A borehole can also be thought to be composed of a sequence of casings and openings. A casing is a section of a borehole with concrete, steel, or plastic installed on the borehole. An opening is a section of a borehole that is open to allow water flow. Additionally, a borehole site may have associated access tubes that provide access to a section of the borehole. Casings, openings, hole interval, and access tubes define the characteristics of a borehole, and the water-level measurements taken at the borehole site are influenced by these aspects.

A primary input data for the ground-water flow model includes discharge (in cubic feet per second) at a springwater site and water depth (in feet below land surface) at a borehole site, both of which are collected by a source agency. Discharge and water depth need to be associated with the time of measurement (in minute). The researchers evaluate the collected water level and discharge measurements to decide which of them will be included as input for the ground-water flow model; the measurements used as an input to the model are referred to as io-water-level (that is, input-output water level) and io-discharge (that is, inputoutput discharge), respectively. There are various hydraulic tests conducted at the borehole site and the results of these tests need to be coupled with the time (in minute) when the test was conducted. Additionally, a borehole site may have a pumplift that removes water from the borehole site; the existence of a pumplift can affect other data collected at the borehole site.

Capturing the data semantics related to, e.g., springwater site, borehole site, borehole, casing, water level, source agency, requires a proposed spatiotemporal conceptual model to:

1. allow the data analyst to model nongeospatial, nontemporal, geospatial, and temporal aspects of the application in a straightforward manner,

2. provide a framework for expressing the structure of spatiotemporal data that is easily understood and communicated to the users,

3. support a mechanism for a methodical translation into implementation-dependent logical models, and

4. include a mechanism to represent various spatial and temporal granularities (e.g., minute, second, degree) in a conceptual schema.

Having summarized some of the requirements for a typical geospatial application, we next describe evaluation criteria for a geospatiotemporal conceptual model designed to capture the data semantics illustrated above. 


\subsection{Evaluation Criteria}

Batini et al. [1] posit that conceptual models should possess the following qualities: expressiveness, simplicity, minimality, and formality. Additionally, to augment extant conventional conceptual models [1], [3], [7] with geospatiotemporal concepts, we need to take into account upward compatibility and snapshot reducibility [2].

Expressiveness refers to the availability of a large variety of concepts for a more comprehensive representation of the real world. Wand et al. [31] posit that "conceptual modeling can be anchored in the models of human knowledge" and that ontology be employed as the basis for a proposed formalism. One of the conflicting goals related to expressiveness is simplicity, which requires that the schema developed using a conceptual model be understandable to both users and data analysts. Prior research [20] contends that one of the deficiencies of the existing conceptual models that can represent geographic phenomena is their inability to "represent information in way that is more natural to humans." While minimality ensures that no concept can be expressed through composition of other concepts, formality specifies that the model must present a unique, precise and well-defined interpretation. Similarly, Wand et al. [32] posit that effective use of conceptual modeling constructs requires that their meaning be defined "rigorously."

Upward compatibility [2] refers to the ability to render a conventional conceptual schema geospatiotemporal without impacting or negating that legacy schema, thus protecting investment in the existing schemas. It also implies that both the legacy schemas and the geospatiotemporal schemas can coexist. Upward compatibility requires that the syntax and semantics of the traditional conceptual model [1], [3], [7] remain unaltered. Snapshot reducibility [2] implies a "natural" generalization of the syntax and semantics of extant conventional conceptual models [1], [3], [7] for incorporating the geospatiotemporal extension. Snapshot reducibility ensures that the semantics of geospatiotemporal model are understandable in terms of the semantics of the conventional conceptual model. Here, the overall objective is to help ensure minimum additional investment in a data analyst training.

Juhn and Naumann [13] posit that conceptual representations "drive discovery" and should be precisely and rigorously defined; on the other hand, discovery needs to be "validated," and the schemas should be clear and comprehensible. In essence, the challenge of adding the space and time dimension is balancing simplicity and understandability with preciseness and completeness.

\section{Ontology-Based Geospatiotemporal Semantics}

Geographic applications require data referenced by geographic coordinates, with time sometimes referred to as the fourth dimension. We summarize key temporal, geospatial, and time-varying geospatial terminology in this section. Next, we describe the annotation syntax and illustrate how ontology is the basis for annotations.

\subsection{Temporal Ontology}

A time domain is denoted by the pair $(T, \leq)$, where $T$ is a nonempty set of time instants and " $\leq$ " is a total order on $T$. We assume that the time domain is discrete (as the measurements modeled are captured at a time known to a discrete value). For example, $(\mathbf{Z}, \leq)$ represents a discrete time domain where instants are denoted by integers, implying that every instant has a unique successor. An instant is a point on the time line. The time between two instants is referred to as a time period. An unanchored contiguous portion of the time line is called a time interval, e.g., one day (or Gregorian day). Unlike time periods, a time interval is a directed duration of time with "no specific starting or ending instants" [11]. A nondecomposable time interval of fixed minimal duration is referred to as a chronon. A finite union of nonoverlapping time periods is referred to as a temporal element [8].

A temporal granularity-an integral part of the temporal data-is defined as a mapping TG from index $i$ to subsets of the time domain [6]. Although the index of a temporal granularity is constrained to be contiguous, the granules are not constrained to be contiguous on the time domain. Thus, a temporal granularity defines a countable set of nondecomposable granules $T G(i)$. Additionally, a special granule called the origin, $T G(0)$, is nonempty. Some examples of temporal granularities are Gregorian-day (with each such granule composed of a sequence of 24 contiguous Gregorianhour granules, or 86,400 contiguous Gregorian-second granules), business-day and business-week (with each such granule composed of five Gregorian-day granules). While Gregorian-day is a temporal granularity with contiguous granules of day, business-day contains some noncontiguous granules (e.g., Friday is followed directly by Monday). Each nonempty granule may have a textual representation referred to as a label (e.g., "2001-10-5 EST"), which can be mapped to an index integer with label mapping. A designated point of time is referred to as an anchor with respect to the time domain. The union of granules is called an image of a temporal granularity.

Facts can interact with time in two orthogonal ways resulting in transaction time and valid time [28]. Valid time denotes when the fact is true in the real world and implies the storage of histories related to facts. Existence time, which applies to an object, is the valid time when the object exists. On the other hand, transaction time links an object to the time it is current in the database and implies the storage of versions of a database object. While the temporal granularity can be specified for existence time and valid time, that for transaction time is system-defined. Time-varying data may be modeled as an event or a state. An event occurs at a point of time, i.e., an event has no duration. A state has duration, e.g., a storm occurred from 5:07 PM to 5:46 PM.

\subsection{Geospatial Ontology}

Any data that can be associated to a location on the earth is referred to as geographic data. A space domain may be represented as a set (e.g., $\mathbf{R}^{3}, \mathbf{R}^{2}, \mathbf{N}^{3}, \mathbf{N}^{2}$ ) with elements referred to as points. For geographic applications, horizontal space is segregated from vertical space; correspondingly, we define horizontal and vertical geospatial granularities [16]. Intuitively, the horizontal space domain corresponds to the earth's surface while the vertical space domain corresponds to the depth/height below/above the sea level. We define a horizontal geospatial granularity as a mapping from integers to any partition of the horizontal space; the partition may arise from pixellation of space and may be a regular square or any other shape like triangular irregular network (TIN) or even irregular shapes (e.g., county). Examples of horizontal geospatial granularities are dms-deg and dms-min.

A geospatial object is associated with position and geometry. The position in space is based on the coordinates 


\begin{tabular}{|c|c|c|}
\hline$\langle$ annotation〉 & $::=$ & $\begin{array}{l}\epsilon \mid\langle\text { temporal annotation }\rangle / /\langle\text { spatial annotation }\rangle \\
\mid\langle\text { temporal annotation }\rangle / /\langle\text { spatial annotation }\rangle / /\langle\text { time-varying spatial annotation }\rangle\end{array}$ \\
\hline$\langle$ temporal annotation $\rangle$ & $::=$ & $€ \mid\langle$ valid time $\rangle /\langle$ transaction time $\rangle$ \\
\hline$\langle$ valid time $\rangle$ & $::=$ & $\langle$ state $\rangle\left(\left\langle\mathrm{g}_{\mathrm{t}}\right\rangle\right) \mid\langle$ indeterminate state $\rangle\left(\left\langle\mathrm{g}_{\mathrm{t}}\right\rangle\right) \mid\langle$ event $\rangle\left(\left\langle\mathrm{g}_{\mathrm{t}}\right\rangle\right) \mid\langle$ indeterminate event $\rangle\left(\left\langle\mathrm{g}_{\mathrm{t}}\right\rangle\right) \mid-$ \\
\hline$\langle$ transaction time $\rangle$ & $::=$ & T|- \\
\hline$\langle$ state $\rangle$ & $::=$ & S | State \\
\hline$\langle$ indeterminate state $\rangle$ & $::=$ & $\langle$ state $\rangle \sim \mid\langle$ state $\rangle+-$ \\
\hline$\langle$ event $\rangle$ & $::=$ & E Event \\
\hline$\langle$ indeterminate event $\rangle$ & $::=$ & $\langle$ event $\rangle \sim \mid\langle$ event $\rangle+-$ \\
\hline$\langle$ spatial annotation $\rangle$ & $::=$ & $\epsilon \mid\langle$ horizontal geometry $\rangle /\langle$ vertical geometry $\rangle$ \\
\hline$\langle$ horizontal geometry〉 & $::=$ & $\langle$ geometry $\rangle\left(\left\langle\mathrm{g}_{x y}\right\rangle\right) /\langle$ geometry $\rangle\left(\left\langle\mathrm{g}_{\mathrm{xy}}\right\rangle\right)$ \\
\hline$\langle$ vertical geometry $\rangle$ & $::=$ & $\langle$ geometry $\rangle\left(\left\langle\mathrm{g}_{z}\right\rangle\right) \mid-$ \\
\hline$\langle$ geometry & $::=$ & $\begin{array}{l}\langle\text { point }\rangle \mid\langle\text { indeterminate point }\rangle \mid\langle\text { line }\rangle \mid\langle\text { indeterminate line }\rangle \mid\langle\text { region }\rangle \\
\mid\langle\text { indeterminate region }\rangle \mid\langle\text { user defined }\rangle \mid-\end{array}$ \\
\hline$\langle$ point $\rangle$ & $::=$ & P|Point \\
\hline$\langle$ indeterminate point $\rangle$ & $::=$ & $\langle$ point $\rangle \sim \mid\langle$ point $\rangle+-$ \\
\hline$\langle$ line $\rangle$ & $::=$ & L| Line \\
\hline$\langle$ indeterminate line $\rangle$ & $::=$ & $\langle$ line $\rangle \sim \mid\langle$ line $\rangle+-$ \\
\hline$\langle$ region $\rangle$ & $::=$ & R|Region \\
\hline$\langle$ indeterminate region $\rangle$ & $::=$ & $\langle$ region $\rangle \sim \mid\langle$ region $\rangle+-$ \\
\hline$\langle$ time-varying spatial annotation $\rangle$ & $::=$ & $\epsilon \mid\langle$ position varying $\rangle \mid\langle$ shape varying $\rangle \mid\langle$ position varying $\rangle /\langle$ shape varying $\rangle$ \\
\hline$\langle$ position varying $\rangle$ & $::=$ & $\langle$ position〉@〈varying in dimension $\rangle$ \\
\hline$\langle$ shape varying $\rangle$ & $::=$ & $\langle$ shape $\rangle @\langle$ varying in dimension $\rangle$ \\
\hline$\langle$ position $\rangle$ & $::=$ & Pos | Position \\
\hline$\langle$ shape $\rangle$ & $::=$ & Sh |Shape \\
\hline$\langle$ varying in dimension $\rangle$ & $::=$ & $x|y| z|x y| y z|x z| x y z$ \\
\hline$\left\langle\mathrm{g}_{\mathrm{t}}\right\rangle$ & $::=$ & $\langle$ day $\rangle \mid\langle$ hour $\rangle \mid\langle$ minute $\rangle \mid\langle$ second $\rangle \mid\langle$ user defined $\rangle$ \\
\hline$\langle$ day $\rangle$ & $::=$ & day \\
\hline$\langle$ hour $\rangle$ & $::=$ & hr|hour \\
\hline$\langle$ minute $\rangle$ & $::=$ & $\min \mid$ minute \\
\hline$\langle$ second $\rangle$ & $::=$ & sec $\mid$ second \\
\hline$\left\langle\mathrm{g}_{\mathrm{xy}}\right\rangle$ & $::=$ & $\langle$ dms-degree $\rangle \mid\langle$ dms-minute $\rangle \mid\langle$ user defined $\rangle$ \\
\hline$\left\langle\mathrm{g}_{\mathrm{z}}\right\rangle$ & $::=$ & $\langle$ mile $\rangle \mid\langle$ foot $\rangle \mid\langle$ user defined $\rangle$ \\
\hline$\langle$ mile $\rangle$ & $::=$ & mile \\
\hline$\langle$ dms-degree $\rangle$ & $::=$ & deg | degree | dms-deg |dms-degree \\
\hline$\langle$ dms-minute $\rangle$ & $::=$ & dms-min |dms-minute \\
\hline$\langle$ foot $\rangle$ & $::=$ & $\mathrm{ft} \mid$ foot \\
\hline
\end{tabular}

Fig. 1. Annotation syntax in BNF.

in a reference system, e.g., latitude and longitude. Geometry represents the shape of an object: a point, a line, and a region. A point is "a zero-dimensional geospatial object with coordinates," a line is "a sequence of ordered points, where the beginning of the line may have a special start node and the end a special end node," and a region consists of "one outer and zero or more inner rings" [30]. The difference between a line and a region is that the line itself is the carrier of information, while the area is of primary importance for a region.

\subsection{Time-Varying Geospatial Ontology}

In geography, space is indivisibly coupled with time. Three types of interaction between an object and space-time are possible [29]:

1. moving objects, i.e., objects whose position changes continuously but the shape does not (e.g., a car moving on a road network),

2. objects whose geospatial characteristics and position change with time discretely, i.e., changing shape (e.g., a change in the shape of land parcels for a cadastral application), and

3. integration of the above two behaviors, i.e., continuous moving and changing phenomena (e.g., modeling a storm).

Having summarized the temporal, geospatial, and timevarying geospatial semantics that need to be captured in a geospatiotemporal conceptual model, we describe how ontology manifests into annotations.

\subsection{Geospatiotemporal Annotations}

Annotations provide a mechanism to specify the context ("when" and "where") associated with "what" is important in the real world. As shown in Fig. 1, the overall structure of an annotation phrase is:

$\langle$ temporal annotation $\rangle / /$ geospatial annotation $\rangle / /$

$\langle$ time-varying geospatial annotation $\rangle$. 


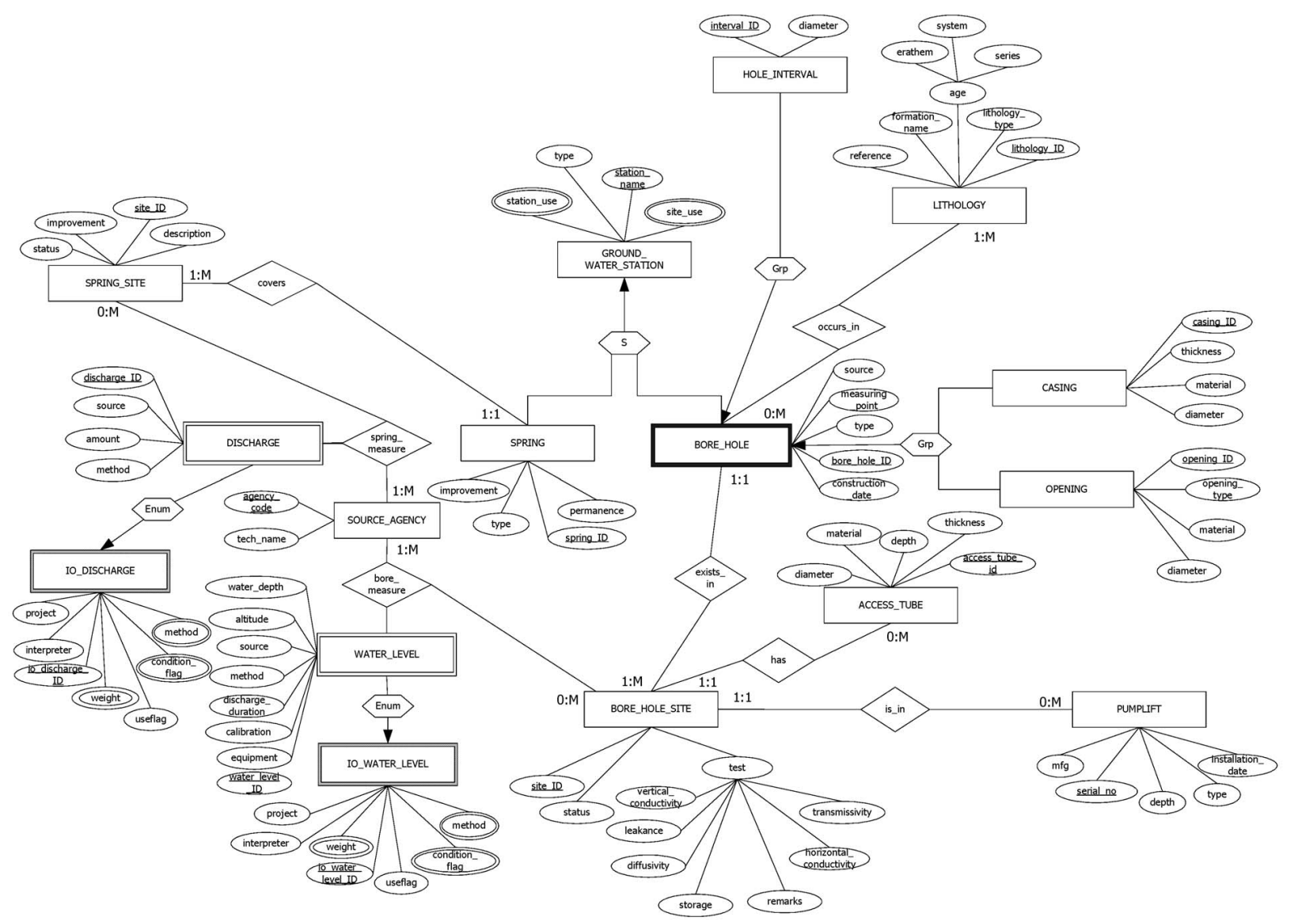

Fig. 2. The USM schema for ground-water flow model.

The temporal annotations, geospatial annotations, and timevarying geospatial annotations are each separated by a double forward slash (//).

The temporal annotation first specifies existence time (or valid time) followed by transaction time. The temporal annotation for valid time and transaction time is segregated by a forward slash (/). Any of these aspects can be specified as not being relevant to the associated conceptual construct by using "-." Valid time or existence time can be modeled as an event (E) or a state (S) and has an associated temporal granularity. For example, "S(min)/T//" associated with DISCHARGE denotes that DISCHARGE exists in a bitemporal space and that the temporal granularity of the states (S) is minute ( $\mathrm{min}$ ). Additionally, we also need to capture transaction time (T) associated with DISCHARGE. In this example, the granularity associated with transaction time is not specified, as it is system-defined.

The geospatial annotation includes geometry and position in $x, y$, and $z$-dimension, and each dimension is segregated by a forward slash (/). For example, "// $\mathrm{P}(\mathrm{deg})$ / P(deg) / -" for SPRING_SITE describes a geometry of points $(\mathrm{P})$ in the $x-y$ plane. The associated horizontal geospatial granularity is degree.

The interaction between an object and space-time can result in a change in the shape and / or a change in the position of an object. A time-varying geospatial annotation can be specified only if geospatial and temporal annotation have already been specified. For example, a moving car tracked by satellite may be represented by an annotation phrase " $E(\mathrm{sec}) /$ - // P(deg) / P(deg) / - // Pos@ $x^{\prime \prime}$ that denotes a time-varying position and a time-invariant shape. The geometry is a point $(\mathrm{P})$ in the $x-y$ plane with geospatial granularity of degree. The position changes in the $x-y$ plane (Pos@xy) over time and each geometry is valid for time granules $(E)$ measured in second. Our annotation also includes a formalism to model indeterminacy; details related to modeling indeterminacy can be found elsewhere [16].

Having outlined geospatiotemporal annotations, we next apply our annotation-based approach to a conventional conceptual model, USM, to propose a geospatiotemporal conceptual model called ST USM. However, our annotation-based approach is not specific to USM and can be applied to any conventional conceptual model [3], [7]. In the next two sections, we exemplify our geospatiotemporal conceptual modeling methodology via USM and ST USM.

\section{USM: REPRESENTING "WHAT"}

The abstractions supported by typical conventional conceptual models [1], [3], [7], [22], [27] include classification, association, aggregation, and generalization/specialization. The underlying principle of these abstractions is selective emphasis of detail. We summarize below the data semantics that can be elicited using conventional conceptual modeling, specifically USM. Fig. 2 illustrates a USM schema that represents "what" is important for the hydrogeologic application described in Section 2.

All real-world objects are referred to by the term entity. Characteristics or properties of entities are called attributes $\left(A_{i}\right.$, where $\left.i=1, \ldots, n\right)$. Each attribute has an attribute domain 
$\left(\operatorname{dom}\left(A_{i}\right)\right)$, which is the set of values that an entity can take for the attribute. An entity class (or class) may be defined as $E=\cup_{i}\left(A_{i}, \operatorname{dom}\left(A_{i}\right)\right)$. The set of instantiations of an entity class is referred to as an entity set. In other words, an entity $e$ of an entity class $E$ may be designated as $e(E)$ and a set of entities of an entity class is represented as $S(E)$, where $e(E) \in S(E)$. For example, in Fig. 2, PUMPLIFT is an entity class which has attributes like serial number (serial_no), manufacturer (mfg), type, and installation date (installation_date).

An interaction relationship refers members of one entity class to members of one or more entity classes. Formally, let $R$ be an interaction relationship and $E_{1}, E_{2}, \ldots, E_{n}$ be classes that participate in the relationship. A relationship may be considered to be a subset of the Cartesian product $S\left(E_{1}\right) \times$ $S\left(E_{2}\right) \times \ldots \times S\left(E_{n}\right)$, where a relationship instance $r_{i}$ consists of exactly one entity from each participating entity set. For example, in Fig. 2, is_in is an interaction relationship between BORE_HOLE_SITE and PUMPLIFT. Each instance of is_in includes an entity from $S$ (BORE_HOLE_SITE) and an entity from $S$ (PUMPLIFT). Note that is_in does not include any interaction attributes and does not have an associated interaction class. The interaction relationship spring_measure includes interaction attributes source, amount, and method, which are represented as attributes of an interaction class DISCHARGE.

Generalization is a form of abstraction in which similar objects are related to a higher-level generic object. For example, in Fig. 2, a GROUND_WATER_STATION is a superclass with SPRING and BORE_HOLE as its subclasses. Certain common attributes in a GROUND_WATER_STATION apply to both SPRING and BORE_HOLE: station_name, site_use, station_use, and type. Attributes like permanence and improvement are specific to SPRING. On the other hand, construction_date and measuring_point are attributes that are associated with BORE_HOLE.

A composite relationship defines a new class called a composite class that has another entity set (or subsets of an entity set) as its members. For example, IO_DISCHARGE is a composite class with DISCHARGE as its component class. Note that the component class DISCHARGE is both a subclass and a subtype of the composite class IO_DISCHARGE.

The grouping establishes a "part-of" or "property-of" relationship. For example, BORE_HOLE is a grouping class with CASING and OPENING as its component classes. Unlike IO_DISCHARGE (a composite class), BORE_HOLE (a grouping class) is not of the same type as CASING or OPENING (component classes).

In this section, we briefly described the semantics associated with a conventional conceptual model, USM. We next explicate the semantics of annotation-based ST USM using USM and constraints in first-order logic.

\section{ST USM: REPRESENTING "WHEN" AND "WHERE"}

Using an example of a temporal entity class, we show the detailed semantics of an annotated abstraction. Details related to other abstractions like attribute, interaction relationship, subclass, composite class, and grouping class are similar to those of a temporal entity class and described elsewhere [15]. The goal is to provide here the essence of our approach.

\subsection{Entity Class}

An application may require capturing the lifespan or transaction time of an entity, the shape and position of an entity in space, or a change in the shape and/or position of an entity over its lifespan, resulting in a temporal entity class, a geospatial entity class, or a time-varying geospatial entity class, respectively.

\subsubsection{Temporal Entity Class}

In a temporal entity class, the membership of an entity in the entity set is time-varying. We assume that a temporal entity class itself (as contrasted with entities of that class) exists during the entire modeled time. Thus, the existence time represents the lifespan of an entity and defines the time when facts associated with an entity can be true in the miniworld. Similarly, we can capture the transaction time associated with an entity, which may be important for applications requiring traceability.

A temporal entity class, e.g., PUMPLIFT, with existence time is associated with an existence time predicate $\varphi_{\text {PUMPLIFT,et }}$ that defines the lifespan of a pumplift in terms of an existence time granularity $T G_{\text {PUMPLIFT,et }}$ (e.g., day). $\varphi_{\text {PUMPLIFT,et }}: S($ PUMPLIFT $) \times \mathbf{Z} \rightarrow \mathbf{B}$. This predicate takes a particular pumplift entity and a particular granule (denoted by an integer; here, a specific day) of the granularity and evaluates to a Boolean that is true if that entity exists in the modeled reality at that granule (day). There are two constraints on the existence time predicate:

1. $\forall e \in S($ PUMPLIFT),

$$
\begin{aligned}
& \forall i, \varphi_{\text {PUMPLIFT,et }}(e, i) \Rightarrow \\
& \left(T G_{\text {PUMPLIFT,et }}(i) \subseteq \operatorname{Image}\left(T G_{\text {PUMPLIFT,et }}\right)\right) .
\end{aligned}
$$

2. $\forall e \in S$ (PUMPLIFT), $\exists i \in \mathbf{Z}, \varphi_{\text {PUMPLIFT,et }}(e, i)$.

The first constraint states that a pumplift can exist only within the defined image of the granularity. Second, every entity exists at some granule (e.g., "2001-7-01") within the image of the granularity. Intuitively, if a pumplift with an associated lifespan does not exist during any granule within the image, it is meaningless to store it in the database. We define an existence temporal projection operator $\left(\pi^{\mathrm{et}}\right)$ as a function that takes a temporal entity and returns the associated temporal element.

Similarly, a temporal entity class PUMPLIFT with transaction time is associated with a transaction time predicate $\varphi_{\text {PUMPLIFT, tt }}$ that defines the transaction time of a pumplift in terms of a transaction time granularity $T G_{\mathrm{tt}}$ (e.g., second). The transaction time granularity is defined only for all points less than now. If a transaction timestamp includes Until Changed (UC, a special transaction time marker), it denotes that the associated fact is current in the database. Unlike the existence time granularity, which can be specified by users, the transaction time granularity is system-defined. $\varphi_{\text {PUMPLIFT,tt }}: S($ PUMPLIFT $) \times\{\mathbf{Z} \cup U C\}$ $\rightarrow$ B. The constraints on the transaction time predicate are similar to those on the existence time predicate. In the same way, a bitemporal entity class PUMPLIFT is associated with $\varphi_{\text {PUMPLIFT, et }}$ and $\varphi_{\text {PUMPLIFT,t }}$ defined in terms of 


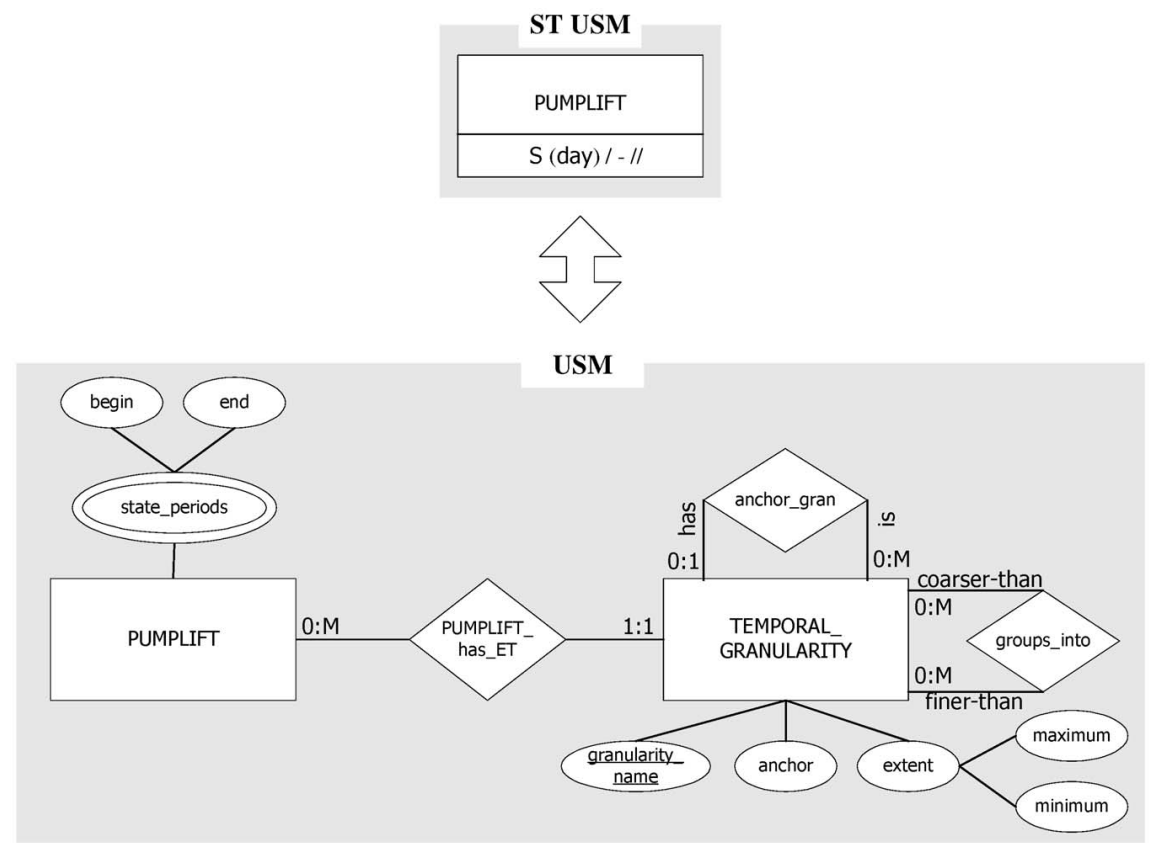

Fig. 3. Temporal entity class in ST USM and its semantics in USM.

an existence time granularity $T G_{\text {PUMPLIFT,et }}$ and a transaction time granularity $T G_{\mathrm{tt}}$, respectively.

Having defined temporal entity class abstractly, we next describe its semantics using an example of PUMPLIFT. When an entity class is defined as temporal, it implies that the application would have queries like: What is the average monthly power consumption by all pumplifts over their installed existence? What are the pumplifts that were installed before 1995 and are operational now? Fig. 3 illustrates the representation of existence time expressed as state (S) with day as the temporal granularity name. The data analyst simply annotates PUMPLIFT, based on the users' requirements, with "S(day)/-//" (as shown in the top part of the figure) and does not need to contend with the complexity of the underlying semantics (shown in the bottom part of the figure) or of the associated temporal constraints. Fig. 3 presents the semantics of a temporal entity class in ST USM via a mapping using the concepts of a conventional conceptual model, which we refer to as a translated USM schema. Note how the spatiotemporal semantics encapsulated via annotations in the ST USM schema are "unpacked" in the translated USM schema. This rendition from an ST USM schema to a (translated) USM schema is snapshot equivalent, that is, the two schemas (ST USM and translated USM) represent the same information content over snapshots taken at all times.

In order to express the semantics of a temporal entity class, we need to specify a TEMPORAL_GRANULARITY in which the evolution of a temporal object is embedded. The relationship PUMPLIFT_has_ET associates an entity with a corresponding TEMPORAL_GRANULARITY. Each TEMPORAL_GRANULARITY is uniquely identified by a granularity_name, shown by an underlined attribute. An extent is the smallest time interval that includes the image of the granularity and is expressed by two indexes, minimum and maximum. Each anchor_gran is a recursive relationship (i.e., a relationship where an entity from the same entity class can play different roles) such that each participating granularity optionally has an anchor $(0: 1)$ and each granularity is an anchor for potentially many other granularities (0:M).

The anchor of a granularity $T G$ is the first index of a strictly finer granularity that corresponds to the origin of this granularity, i.e., $T G(0)$. All granularities except the bottom granularity have an associated anchor. A finer-than and a coarser-than relationship between granularities are denoted by a recursive relationship groups_into, where one granularity plays the role of finer-than and the other the role of coarser-than. The relationships anchor_gran together with groups_into help create a granularity graph [6], which can aid a designer in choosing the level of detail associated with facts. Details related to granularities and indeterminacy vis-à-vis ST USM are presented elsewhere [16].

A temporal entity with existence time may have a set of event_instants or state_periods associated with it, depending on whether a temporal entity is represented as an event or a state. A time period of PUMPLIFT is represented with indexes begin and end of state_periods. A double-lined ellipse in USM denotes a multivalued attribute. For example, state_periods is represented as a multivalued attribute and represents a set of state periods (i.e., a temporal element) associated with an entity.

We now describe the constraints on temporal entities of PUMPLIFT. These inherent constraints in the ST USM schema are rendered as explicit constraints in the translated USM schema. Constraints 5.1.1 and 5.1.2 are based on our definition of a temporal entity, i.e., a temporal entity has an associated temporal granularity and has an associated temporal element. Constraints 5.1.3-5.1.5 are based on the definition of a temporal element. In these definitions, we assume a closed-open representation, i.e., the begin index is contained in the period while the index corresponding to the end is not. For example, an instant for a temporal element may be represented by $[17,18)$. In this example, begin index (i.e., 17) is inclusive in the instant while end index (i.e., 18) is not. 
Constraint 5.1.1: The existence time for all the entities of PUMPLIFT have the same associated granularity; in this case, day.

$\forall e \in S(\mathrm{PUMPLIFT})$, e.PUMPLIFT_has_ET.TEMPORAL_GRANULARITY (granularity_name) $=$ day.

Constraint 5.1.2: Every entity of PUMPLIFT has an associated temporal element with well-formed periods.

$\forall e \in S$ (PUMPLIFT), $\exists p \in e$.state_periods, $p$.begin $<p$.end.

Constraint 5.1.3: State periods of an entity of PUMPLIFT are well-formed.

$\forall e \in S$ (PUMPLIFT), $\forall p \in e$.state_periods, $p$.begin $<p$.end.

Constraint 5.1.4: Temporal elements are well-formed. A temporal element is defined as a union of nonoverlapping time intervals.

$$
\begin{gathered}
\forall e \in S(\text { PUMPLIFT }), \forall p_{1}, p_{2} \in e . \text { state_periods }, \\
p_{1} \text {.begin }<p_{2} . \text { begin } \Rightarrow p_{1} \text {.end } \leq p_{2} \text {. begin. }
\end{gathered}
$$

Constraint 5.1.5: The extent of a temporal granularity defines the upper and lower bounds for any temporal element. In other words, a temporal element cannot include an index that is larger than the corresponding extent.maximum or smaller than the corresponding extent.minimum.

$\forall e \in S$ (PUMPLIFT), $\forall p \in$ e.state_periods,

$$
\begin{aligned}
& \text { e.PUMPLIFT_has_ET.TEMPORAL_GRANULARITY } \\
& \text { (extent.minimum) } \leq p \text {. begin }<p . \text { end } \leq \\
& \text { e.PUMPLIFT_has_ET.TEMPORAL_GRANULARITY } \\
& \text { (extent.maximum). }
\end{aligned}
$$

Constraints 5.2.1-5.2.3 are based on the definition of a temporal granularity. These constraints will be generated once for the entire schema.

Constraint 5.2.1: Each TEMPORAL_GRANULARITY has a lower and an upper bound referred to as minimum and maximum; these bounds are well-formed.

$$
\begin{aligned}
& \forall e \in S(\text { TEMPORAL_GRANULARITY }), \\
& \quad e(\text { extent.minimum })<e(\text { extent.maximum }) .
\end{aligned}
$$

Constraint 5.2.2: All the granularities, except one, have an anchor. In other words, the bottom granularity is allowed not to have an anchor.

$$
\begin{aligned}
\forall e_{1} \in S(\text { TEMPORAL_GRANULARITY }), \\
\neg \text { has }\left(e_{1} \text {.anchor_gran }\right) \Rightarrow \\
\neg\left(\exists e_{2} \in S(\text { TEMPORAL_GRANULARITY })\right. \\
\left.\wedge e_{1} \neq e_{2} \wedge \neg \text { has }\left(e_{2} \text {.anchor_gran }\right)\right) .
\end{aligned}
$$

Constraint 5.2.3: For a temporal granularity, if an anchor does not exist then that is the bottom granularity that does not have any granularity finer than it; in other words, it cannot take the role of coarser-than in the relationship groups-into.

$$
\begin{aligned}
\forall e \in S & (\text { TEMPORAL_GRANULARITY }), \\
& \neg \text { has }(e \text {.anchor_gran }) \Rightarrow \\
& \neg \text { coarser-than(e.groups_into }) .
\end{aligned}
$$

As exemplified by an example of PUMPLIFT, the temporal annotation associated with an entity class renders it sequenced, i.e., the entity exists for each point in time (specified by granularity indexes) within the specified lifespan. Note how the annotation phrase (e.g., "S(day)/-//") associated with an entity class encapsulates the semantics that are explicated in Fig. 3 and Constraints 5.1.1-5.1.5 and 5.2.1-5.2.3. As may be evident, an easily-expressed annotation phrase may represent quite complex semantics.

\subsubsection{Geospatial Entity Class}

A geospatial entity class refers to georeferenced entities with an associated shape and position, which is used to locate them in a two or three-dimensional space. In this section, we first define a geospatial entity in terms of a geospatial granularity and then describe the associated semantics of a geospatial entity class in ST USM, using examples of SPRING_SITE and BORE_HOLE_SITE.

A geospatial entity in a horizontal space domain, e.g., SPRING_SITE, is associated with a horizontal geometry predicate $\Psi_{\text {SPRING_SITE,xy }}$ that defines the location of a spring site in terms of horizontal geospatial granularity. $\Psi_{\text {SPRING_SITE,xy }}: S($ SPRING_SITE) $\times \mathbf{Z} \rightarrow \mathbf{B}$.

Spatial partitions-formed in a 2 or 3-dimensional space-are represented by integers. Constraints on horizontal geometry predicate are similar to those on the existence time predicate. We can define a horizontal geospatial projection operator $\left(\pi^{\mathrm{xy}}\right)$ that takes a geospatial entity and returns its geometry (point, line, or region). In the case of SPRING_SITE, $\pi^{\mathrm{xy}}$ is constrained to be a point on the horizontal surface.

A geospatial entity in 3-dimensional space, e.g., BORE_ HOLE_SITE, is associated with $\Psi_{\text {BORE_HOLE_SITE,xy }}$ and $\Psi_{\text {BORE_HOLE_SITE,z }}$ that defines the location of an entity in terms of horizontal and vertical geospatial granularities, i.e., $S G_{\text {BORE_HOLE_SITE,xy }}$ and $S G_{\text {BORE_HOLE_SITE,z, respectively. }}$ For an entity in 3-dimensional space, we define a vertical geospatial projection operator, $\pi^{\mathrm{z}}$. In the example above, $\pi^{\mathrm{xy}}$ is constrained to be a point and $\pi^{\mathrm{z}}$ is constrained to be a line.

The semantics of a geospatial entity class can be defined like those for a temporal entity class described in the previous section [15].

\subsubsection{Time-Varying Geospatial Entity Class}

A time-varying geospatial entity class models two types of changes: 1) Nongeospatial change refers to an entity with a fixed associated position/geometry and a lifespan, and 2) geospatial change denotes an entity whose geometry varies over its lifespan. While the former models a geospatial entity with an associated existence time (and/or transaction time), the latter captures a change in shape and/or position over time (existence time and/or transaction time) [29]. For the second 
TABLE 1

The Semantics of Temporal/Nontemporal Attribute/Entity Combinations

\begin{tabular}{l|ll} 
& Non-temporal Attribute & Temporal Attribute \\
\hline $\begin{array}{l}\text { Non-temporal Entity } \\
\text { Class }\end{array}$ & $\begin{array}{l}\text { Only the current properties of } \\
\text { the currently relevant entities }(I)\end{array}$ & $\begin{array}{l}\text { Maintain attribute value } \\
\text { histories of the currently } \\
\text { relevant entities (2) }\end{array}$ \\
\hline $\begin{array}{l}\text { Temporal Entity Class/ } \\
\text { Time varying Geospatial } \\
\text { Entity Class }\end{array}$ & $\begin{array}{l}\text { Only the current properties over } \\
\text { the lifespan of entities (3) }\end{array}$ & $\begin{array}{l}\text { Maintain attribute value } \\
\text { histories over the lifespan of } \\
\text { entities (4) }\end{array}$
\end{tabular}

case, only the position of an entity may change continuously while its shape does not (e.g., a transportation application), or the shape may change discretely (e.g., a cadastral application), or both position and shape may change continuously (e.g., modeling a hurricane).

For the first case, a time-varying geospatial entity class is associated with an existence predicate (i.e., $\varphi_{E, \mathrm{et}}$ ), a (horizontal) geometry predicate (i.e., $\Psi_{E, \mathrm{xy}}$ ), and the geospatial projection is not time-varying. Intuitively, this implies that the application needs to capture when and where entities exist. However, queries that involve evolving geometries over time like: "During the year 2001, did the surface area of Lake Mesquite decrease by more than 10 percent of its area measured on 2000-08-25?" are not required of the application. The annotation for this case is simply a combination of the geospatial and temporal annotation already described in the previous two sections.

For the second case above, the application needs to capture various shapes and/or positions of the entities over time. Our annotation syntax includes a formalism to specify whether position (Pos or Position) and/or shape (Sh or Shape) is timevarying. Additionally, the user can also specify the dimension (e.g., xy and xyz) over which the position and/or shape changes over time. However, to include a time-varying geospatial annotation, the temporal and geospatial annotation should already have been specified. The time-varying geospatial annotation is specified after the second double forward slash (//). For example, "S(min)/-// R(dms-sec)/ $\mathrm{R}(\mathrm{dms}-\mathrm{sec}) /-/ / \mathrm{Sh} @ \mathrm{xy}$ " implies that the shape of entities is a region $(\mathrm{R})$ in an $x-y$ plane with a horizontal geospatial granularity of dms-second. The shape of the entity changes over time in the $x$ - $y$ plane (Sh@ $@ x y$ ) and each shape is valid for a set of time granules ( $\mathrm{S} \equiv$ state) measured in minute. If the geometry changes from a region to a point (or even line), the geometry is still generically represented as region $(R)$ only, as a point (or a line) is a degenerate region.

The semantics of a time-varying geospatial entity class can be defined like those for a temporal entity class [15].

\subsection{Attribute}

Entities have properties referred to as attributes that represent facts that need to be captured for a database application. An entity class can have two kinds of attributes, descriptive attributes and geospatial attributes. If the user wants to elicit the evolution of facts over time, the attribute is referred to as a temporal attribute. On the other hand, when the user wants to capture the evolution of geospatial facts over time, the attribute is referred to as a time-varying geospatial attribute.

\subsubsection{Temporal Attribute}

Temporal attributes represent properties of an entity that are associated with valid time and/or transaction time. The temporal annotation for an attribute is the same as that for a temporal entity class described in the previous section. Annotating an attribute renders it sequenced, implying that the property is true for each point in time within the associated temporal element.

As shown in Table 1, a nontemporal or temporal attribute can be associated with a temporal or nontemporal entity class. A temporal entity class implies that objects are pertinent for a database application even when they are not current in the modeled reality. A nontemporal entity class implies that only the currently legitimate objects are important for the database application. If a nontemporal entity is no longer currently legitimate, one does not need to store the evolution of facts associated with such an entity (cell 2 in Table 1). For example, a nontemporal entity class SOURCE_AGENCY with a temporal attribute tech_name would imply that histories of only currently relevant source agencies are pertinent for the application. A nontemporal attribute of a temporal entity (cell 3 in Table 1) indicates that: 1) the attribute does not vary with time, 2) the user is only interested in the last recorded value of the attribute and not in its history, or 3) the time associated with the attribute is unknown. A temporal attribute for a temporal entity class (cell 4 in Table 1) necessitates that the valid time of the attribute is equal to the lifespan of the entity, which is a direct implication from the semantics of a conventional conceptual model where attributes are specified for an existing entity. Cell 1 in Table 1 represents a nontemporal entity class with nontemporal attribute in a conventional conceptual model with implicit snapshot semantics.

Note how snapshot reducibility naturally extends (i.e., cells 2, 3, and 4) the conventional semantics shown in gray (i.e., cell 1).

A single-valued temporal attribute is one where each entity has a maximum of one value for any time granule; however, it can have multiple values over the lifetime of the entity. A temporal attribute $A$ of an entity class $E$ with valid time is associated with an attribute valid time function $\varphi_{E, A, \mathrm{vt}}$ that defines the attribute values, $\operatorname{dom}(A)$, at different time granularity indexes. $\varphi_{E, A, \mathrm{vt}}: S(E) \times \mathbf{Z} \rightarrow 2^{\operatorname{dom}(A)}$. The constraints on the attribute valid time function are described below:

$$
\begin{aligned}
& \text { 1. } \forall e \in S(E), \\
& \quad \varphi_{E, A, \mathrm{vt}}(e, i) \in \operatorname{dom}(A) \Rightarrow\left(T G_{E, A, \mathrm{vt}}(i) \subseteq\right. \\
& \left.\quad \operatorname{Image}\left(T G_{E, A, \mathrm{vt}}\right)\right) .
\end{aligned}
$$

2. $\forall e \in S(E), \forall i, \varphi_{E, \mathrm{et}}(e, i) \Rightarrow \varphi_{E, A, \mathrm{vt}}(e, i) \in \operatorname{dom}(A)$. 
TABLE 2

The Semantics of Geospatial/Nongeospatial Attribute/Entity Combinations

\begin{tabular}{l|ll} 
& Non-Geospatial Attribute & Spatial Attribute \\
\hline $\begin{array}{l}\text { Non-Geospatial Entity } \\
\text { Class }\end{array}$ & Conventional entity/attribute (1) & Space-varying attribute values (2) \\
\hline $\begin{array}{l}\text { Spatial/ Time-varying } \\
\text { Geospatial Entity } \\
\text { Class }\end{array}$ & $\begin{array}{l}\text { Value of the attribute applies to } \\
\text { the entire geometry of the } \\
\text { geospatial entity (3) }\end{array}$ & $\begin{array}{l}\text { Space-varying attribute values } \\
\text { within the geometry of a } \\
\text { geospatial entity (4) }\end{array}$
\end{tabular}

The first constraint states that the history of an attribute can be defined within the image of the granularity of the attribute. The second constraint-which applies when the associated attribute of a temporal entity is also temporal (cell 4 of Table 1) - states that the history of an attribute is defined within the lifespan of the associated entity. If no value is defined for a granularity index where an entity exists, the corresponding value of the temporal (optional) attribute for that index is assumed to be unknown; this is a direct implication from the semantics of a conventional conceptual model where optional attributes [22] imply properties with unknown values. Similar to mandatory attributes that are required to have a nonnull value, temporal mandatory attributes are required to have nonnull values at each point in time (i.e., a sequenced constraint). This is natural generalization of the definition of a mandatory attribute. While a single-valued temporal attribute, $\varphi_{E, A, \mathrm{vt}}(e, i)$ is constrained to be a singleton (i.e., a set with only one element), no such restriction exists for a multivalued temporal attribute.

Similar to a temporal attribute with valid time, a temporal attribute with transaction time is associated with an attribute transaction function $\varphi_{E, A, t \mathrm{t}}$ and a bitemporal attribute is associated with $\varphi_{E, A \text {,tt }}$ and $\varphi_{E, A, \mathrm{vt}}$.

We now describe the constraints on a temporal attribute. The constraints related to a temporal element are similar to Constraints 5.1.3-5.1.5. The granularity-based Constraints 5.2.1-5.2.3 hold for a temporal attribute also. Constraint 5.3.1 is a sequenced consequent of a (nontemporal) USM, where an attribute (e.g., attr) draws values from a domain (e.g., $\operatorname{dom}(a t t r) \cup \mathrm{NULL})$; a temporal attribute draws values from the domain at each point in time during the lifespan of the entity.

Constraint 5.3.1: If both the entity class and its attribute are temporal, the union of the temporal elements of an (temporal) attribute (ap) must be equal to the lifespan of the associated entity (ep).

$$
\begin{aligned}
& \forall e \in S(\langle\text { ENTITY_CLASS }\rangle), \forall e p \in e . s t a t e \_p e r i o d s, \\
& \quad \forall k \in[e p . \text { begin, } e p . e n d), \\
& \quad \exists a \in e .\langle\text { ENTITY_CLASS }\rangle \text { _tt-attrib }\rangle \text { REL. } \\
& \quad\langle\text { ENTITY_CLASS }\rangle\langle\langle\text { t-attrib }\rangle, \\
& \quad \exists a p \in a . \text { state_periods, ap.begin } \leq k<a p . \text { end. }
\end{aligned}
$$

Identifiers (also called keys) are one or more attributes used to identify members of an entity set. Annotating a key attribute renders it sequenced. In other words, a temporal key-a sequenced constraint-is a uniqueness constraint at each point in time.

Constraint 5.3.2: If 〈t-attrib〉 is a key attribute, there will be an additional uniqueness constraint on this attribute. At each point of time within the temporal element, the number of entities with the same value of the key attribute is 1 .

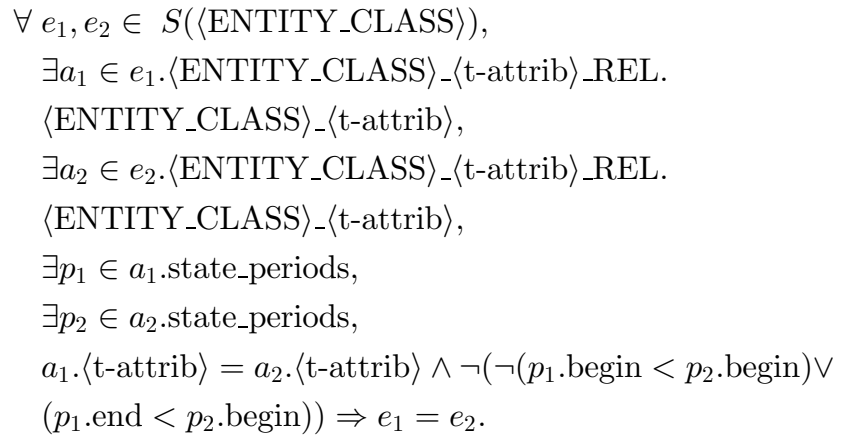

\subsubsection{Geospatial Attribute}

Geospatial attributes represent properties that are georeferenced with respect to the earth. Like temporal annotations, geospatial annotations render the schema sequenced spatially.

As shown in Table 2, a nongeospatial and geospatial attribute can be associated with a nongeospatial entity and geospatial (or time-varying geospatial) entity class.

A geospatial attribute of a geospatial entity class implies that the attribute has different values for different parts within the geometry of the geospatial entity (cell 4 of Table 2). A nongeospatial attribute of a geospatial entity implies that the value of a property applies to the entire geometry of the object (cell 3 of Table 2). For example, status, a nongeospatial attribute of BORE_HOLE_SITE, refers to an entire (geometry of) borehole site. A geospatial attribute of a nongeospatial entity implies a space-varying attribute (cell 2 of Table 2). The annotation syntax for a geospatial attribute is the same as that for a geospatial entity. A geospatial attribute $A$ of an entity class $E$ with geometry is associated with an attribute geometry function $\Psi_{E, A, \mathrm{xy}}$. The semantics associated with geospatial attributes and time-varying geospatial attributes are similar.

\subsection{Interaction Relationship}

An interaction relationship relates members of an entity set to those of one or more entity sets.

\subsubsection{Temporal Relationship}

A temporal relationship implies the need to track the evolution of the interaction between temporal entities in a relationship. For example, if is_in were a temporal relationship between two temporal entity classes BORE_HOLE_SITE and PUMPLIFT, it would imply that an application might include 
TABLE 3

The Semantics of Temporal/Nontemporal Relationship/Entity Class Combinations

\begin{tabular}{l|ll} 
& Non-temporal Relationship & Temporal Relationship \\
\hline $\begin{array}{l}\text { Non-temporal Entity } \\
\text { Class }\end{array}$ & $\begin{array}{l}\text { Currently valid relationship } \\
\text { between currently valid entities (1) }\end{array}$ & N/A (2) \\
\hline Temporal/ Time- & Currently valid relationship among & Temporal relationship among \\
varying Geospatial & temporal (time-varying geospatial) & temporal (time-varying \\
Entity Class & entities (3) & geospatial) entities (4)
\end{tabular}

queries like "In the last six months, what are the various pump lifts associated with the borehole site 12345."

As shown in Table 3, a temporal/nontemporal relationship can be associated with temporal/nontemporal entity class. A temporal relationship can be defined only when all the participating entities are also temporal. This again is a direct implication of the semantics of a relationship in a conventional conceptual model where relationships can only be defined between entities that exist. Thus, a temporal relationship between nontemporal entities (cell 2 of Table 3 ) is not legal in ST USM, as that would imply the existence of a relationship even when the associated entities did not exist.

If the participating entity classes are temporal but the relationship is not (cell 3 of Table 3 ), the entities participating in the relationship should be valid now. If $E_{1}, \ldots, E_{n}$ are temporal entity classes participating in a nontemporal relationship $R, \forall\left(e_{1}, \ldots, e_{n}\right) \in S(R), \varphi_{E 1 \text {,et }}\left(e_{1}, U C\right) \wedge \ldots \wedge$ $\varphi_{E n, \text { et }}\left(e_{n}, U C\right)$. In this case, the temporal element of the relationship is constrained to be a subset $(\subseteq)$ of the temporal elements of the participating entities. Constraint 5.4.1 is a sequenced analog of the USM relationship where a relationship is defined between entities that exist.

Constraint 5.4.1: The temporal element of a temporal relationship is a subset of the intersection of the temporal elements of the participating entities.

$$
\begin{aligned}
& \forall e_{1} \in S\left(E_{1}\right), e_{2} \in S\left(E_{2}\right), \ldots, e_{n} \in S\left(E_{n}\right), \forall\left(e_{1}, e_{2}, \ldots, e_{n}\right) \in \\
& \langle\text { rel }\rangle, \exists p_{1} \in e_{1} \text {.state_periods, } \ldots, \exists p_{n} \in e_{1} \text {.state_periods, } \\
& \forall r p \in\left(e_{1}, e_{2}, \ldots, e_{n}\right) \text {.state_periods, } p_{1} \text {.begin } \\
& \leq \ldots \leq p_{n} \text {. begin } \leq r p \text {.begin }<r p . \text { end } \leq p_{n} \text {.end } \\
& \leq \ldots \leq p_{1} \text {.end. }
\end{aligned}
$$

Other constraints on a temporal relationship are similar to Constraints 5.1.1-5.1.5 and 5.2.1-5.2.3. If the granularity of the relationship is not the same as the granularity of the participating entities, a granularity lattice [6] can be employed to convert the granularities.

The cardinality constraint-a structural constraint-specifies the number of relationship instances that an entity can participate in. As described in Section 4, a cardinality constraint of 1:M associated with a pumplift in the relationship is_in implies that a borehole site can have a minimum of 0 and a maximum of many $(\mathrm{M})$ associated pumplifts. Temporal entities participating in a temporal relationship renders this constraint sequenced and is referred to as the snapshot cardinality constraint. For example, in Fig. 2, it implies that in the relationship is_in, each BORE_HOLE_SITE can have a minimum of 0 and a maximum of many (M) associated PUMPLIFT at each point in time (represented by the granularity index).

\subsubsection{Geospatial Relationship}

A geospatial relationship refers to relationships between the geometries of the participating entities. As shown in Table 4, a geospatial/nongeospatial relationship can be associated with a geospatial/nongeospatial entity class. A geospatial relationship between one or more geospatial (or time-varying geospatial) entities (cell 4 of Table 4 ) specifies an explicit relationship between the geometries of the participating entities. In other words, this implies an association with at least one geospatial projection among the participating entities. For example, occurs_in ("//-/-/L(ft)") is a geospatial relationship between BORE_HOLE and LITHOLOGY.

While LITHOLOGY is a nongeospatial entity class, BORE_HOLE is a geospatial entity class. A geospatial relationship means that different geospatial parts of a borehole (along the $z$-dimension) are associated with different lithologies. Capturing this relationship would enable answering queries like: "What is the lithology at a depth of 50 feet of a specified borehole?" A nongeospatial relationship between geospatial entities (cell 3 of Table 4) implies a relationship among entities that is unrelated to its geometry. A geospatial relationship between nongeospatial entities (cell 2 of Table 4) is illegitimate as it contradicts the definition of a geospatial relationship, i.e., it is a relationship between the geospatial projection of geospatial entities.

In this section, we outlined the semantics of annotations

\begin{tabular}{|c|c|c|}
\hline & Non-Geospatial Relationship & Geospatial Relationship \\
\hline $\begin{array}{l}\text { Non-geospatial Entity } \\
\text { Class }\end{array}$ & $\begin{array}{l}\text { Traditional relationship semantics } \\
(1)\end{array}$ & N/A (2) \\
\hline $\begin{array}{l}\text { At least one Geospatial/ } \\
\text { Time-varying } \\
\text { Geospatial Entity Class }\end{array}$ & $\begin{array}{l}\text { Association among entities; not } \\
\text { related to geometries (3) }\end{array}$ & $\begin{array}{l}\text { Association with at least one } \\
\text { geometry of the participating } \\
\text { entities ( } 4 \text { ) }\end{array}$ \\
\hline
\end{tabular}
and how our annotation-based approach naturally extends the semantics of a conventional conceptual model. Having

TABLE 4

The Semantics of Geospatial/Nongeospatial Relationship/Class Combinations 


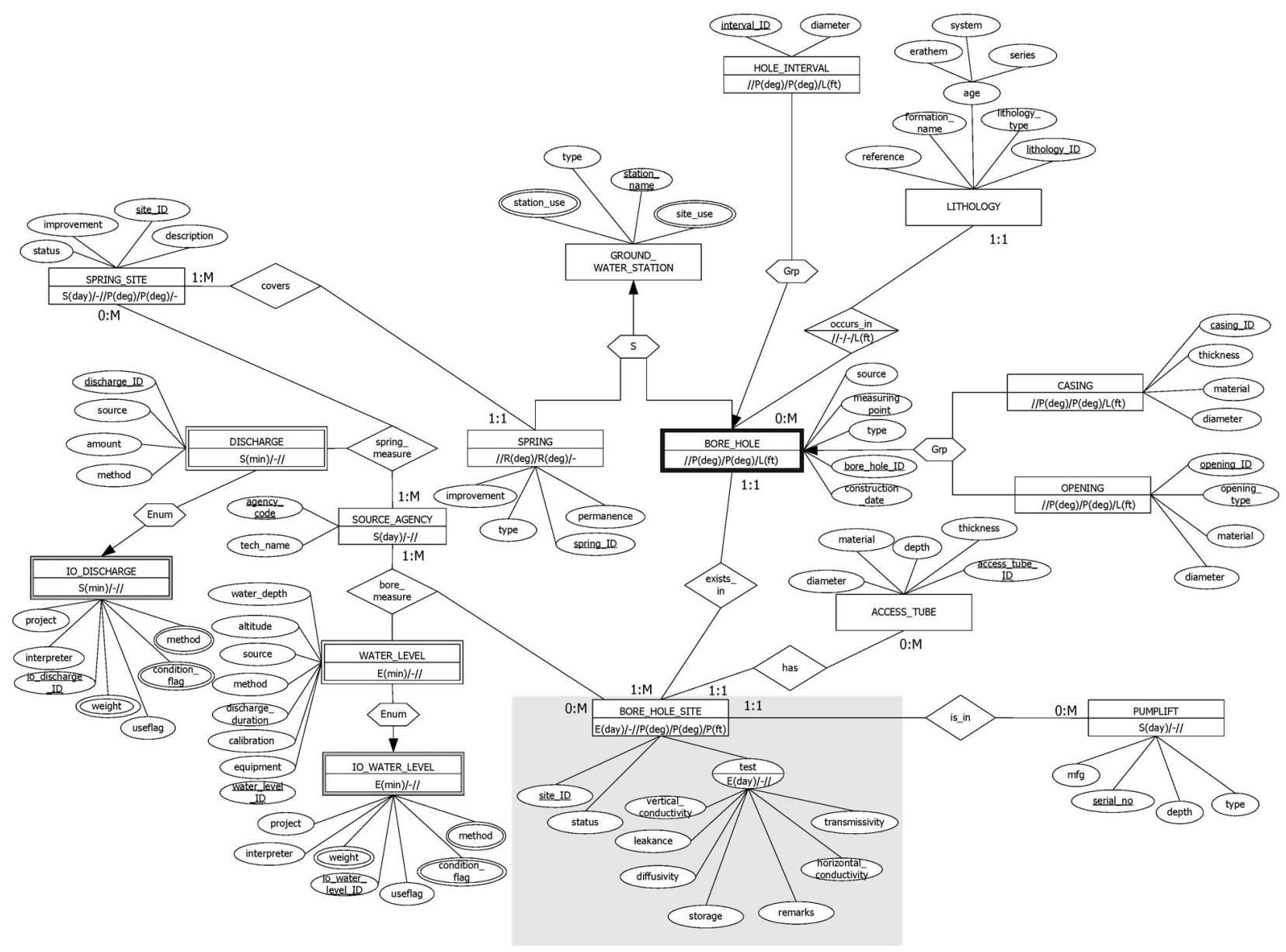

Fig. 4. An annotated schema (ST USM) for the ground-water flow model.

described the syntax (Section 3.4) and semantics (Sections 5.15.3) of a geospatiotemporal conceptual model, we apply our approach to the hydrogeologic application described in Section 2.

\subsection{A Geospatiotemporal Application: Reprise}

Based on the temporal and geospatial requirements described in Section 2, the data analyst captures the geospatiotemporal requirements of the user using annotations. At this time, the data analyst asks the application users questions like: Do you want to store the history or only the current value of this fact? Do you want to capture the history of facts (valid time) or sequence of updates (transaction time), or both? What is the associated temporal granularity? Does the fact need to be modeled as an event or a state? Accordingly, the data analyst annotates the schema shown in Fig. 2 resulting in the annotated schema (or ST USM schema) shown in Fig. 4.

Note how Fig. 4 augments the schema shown in Fig. 2 with geospatiotemporal annotations. For example, SPRING needs to be represented as a region with horizontal geospatial granularity of degree. The annotation phrase associated with the entity class SPRING is "//R(deg)/ $\mathrm{R}(\mathrm{deg}) / . .$.

The attribute test (of entity class BORE_HOLE_SITE) is a temporal attribute represented as an event with temporal granularity of day. A borehole may have different lithology at different depths. While LITHOLOGY is a nongeospatial entity class, BORE_HOLE is a geospatial entity class. The occurs_in relationship is geospatial, associating LITHOLOGY to different depths of BORE_HOLE. which is why the annotation for occurs_in is "//-/-/L(ft)."

Since a translated USM schema for the complete ST USM schema in Fig. 4 would be very involved, we take a small portion of the ST USM schema (the gray portion of Fig. 4) and present the explicated geospatiotemporal semantics via a translated USM schema in Fig. 5. Additionally, 15 constraints-implicit in the ST USM schema in Fig. 4-are associated with the translated USM schema (shown in Fig. 5) [15].

In our geospatiotemporal conceptual design methodology, the annotated schemas capture geospatiotemporal requirements of the users and validate their requirements. While the ST USM schema succinctly encapsulates the geospatiotemporal data semantics, the translated USM schema explicates the geospatiotemporal semantics in terms of the abstractions of a conventional conceptual model and constraints expressed first-order logic. As shown by this example, a few straightforward annotations capture the (quite complex) underlying geospatiotemporal data semantics of the application.

Mapping rules provide correspondences between conceptual and logical model constructs and are applied in 


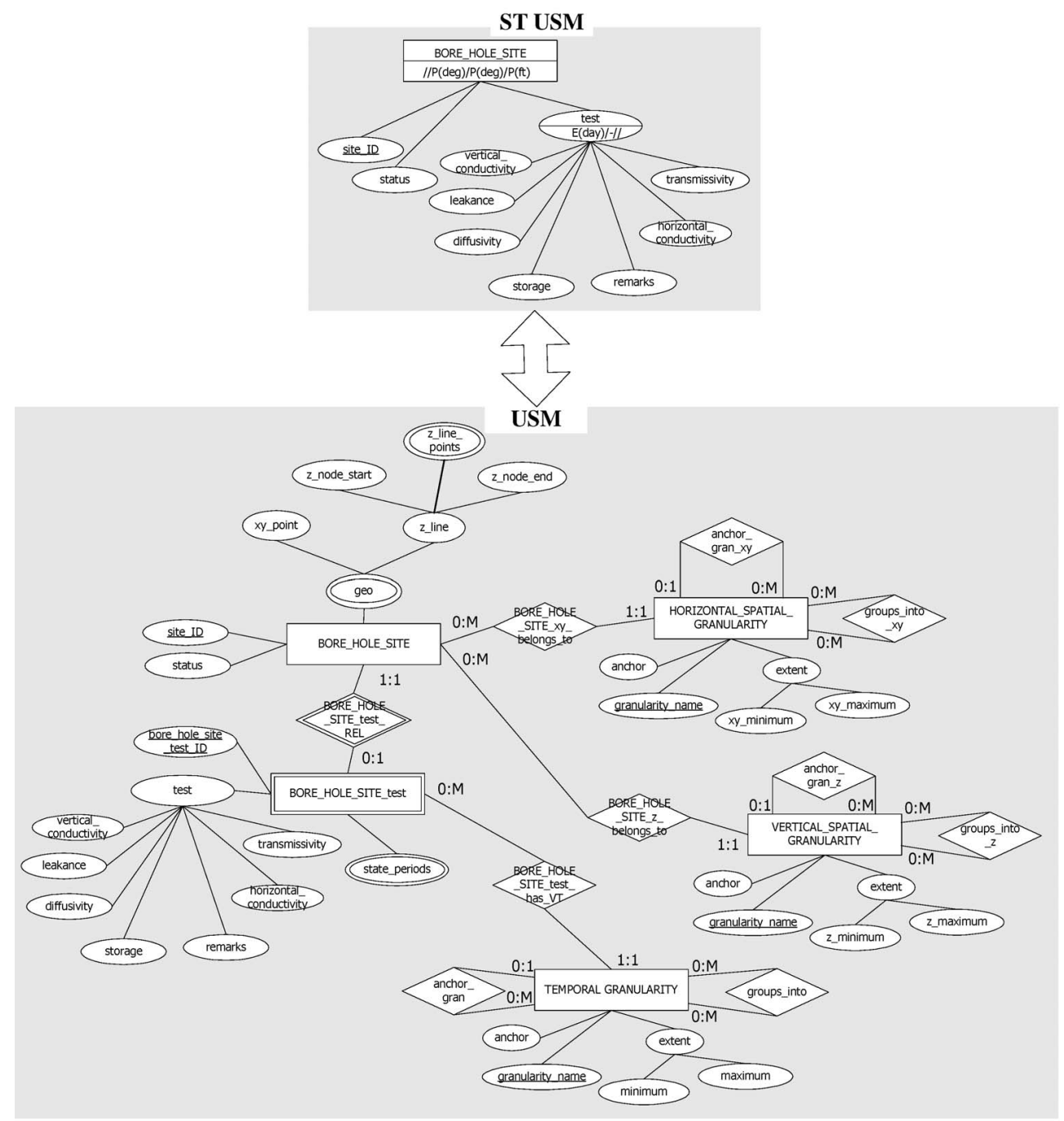

Fig. 5. ST USM schema and its semantics using translated USM schema.

logical design. Such a (logical) mapping depends on the geospatiotemporal support provided by the logical model, which is outside the scope of this paper.

\section{Evaluation}

We evaluate our proposed approach based on the criteria explicated in Section 2.2.

- Expressiveness: We proposed intuitive ontology-based grammar for annotation that comprehensively captures the semantics related to space and time.

- Simplicity: With our approach, we have integrated the semantics of space and time into a traditional conceptual model. Simplicity implies that 1) our approach is generic and can be integrated into any conventional conceptual model [1], [3], [7]; 2) the syntax is straightforward to understand and use, as shown by a separate user study [14]; and 3) our proposed formalism, if adopted into an existing conceptual design tool (e.g., DISTIL [14]), would require minimal changes to that tool.

- Minimality: Since various types of conceptual modeling abstractions (e.g., entity, attribute, relationship, and $k e y$ ) are orthogonal to space and time, the annotations are minimal and generic, i.e., applicable to all types of conceptual modeling abstractions.

- Formality: We have defined the syntax formally in BNF (Fig. 1) and used first-order logic to define the semantics formally (cf. Section 5).

- Upward compatibility: As our proposed extension is a strict superset provided by adding nonmandatory semantics, the geospatiotemporal extension is upward compatible with the conventional conceptual model.

- Snapshot reducibility: Our annotation-based approach naturally extends the conventional conceptual model without increasing complexity from the perspective of users, data analysts, and CASE (ComputerAided Software/Systems Engineering) tool vendors. With our annotation-based approach, we claim to have achieved comprehensiveness and formality along with simplicity in geospatiotemporal conceptual modeling.

\section{SUMMARY}

Lee and Isdale [18] argue that there is a need for a special purpose conceptual model that is suitable for GIS applications. Additionally, the proposed model of space and time 
needs to be reconciled with the extant conceptual models developed in the database community [9].

A data semantics provides "a connection from a database to the real world outside the database" [26], and a conceptual model provides a mechanism to capture the data semantics. In this paper, we described an annotationbased approach for elicitation of the geospatiotemporal semantics. While we posit that the spatiotemporal annotation presented in this paper is comprehensive, it is impossible to assert completeness with conceptual modeling because any formalism is motivated in part by pragmatic rather than purely theoretical reasons. It is possible that the formalism presented in this paper may need to be extended for a geospatiotemporal application, e.g., mobile transactions [12], [25]. In such a case, the annotations presented in this paper can be easily extended. Since spatiotemporal annotations are orthogonal to the conceptual modeling abstractions, our annotation-based approach is not only generic, but also straightforward to extend.

Further work would be useful in several areas. It would also be helpful to explore how ST USM can be used as a canonical model for information integration of distributed geospatiotemporal data. The annotations should be extended to incorporate schema versioning [24], as well as to provide a mechanism for modeling geospatiotemporal constraints in a conceptual schema, such as lifetime constraints and topological constraints. Finally, it will be useful to explore how annotations can be applied to geospatiotemporal processes (STP) [4].

\section{ACKNOWLEDGMENTS}

This work was supported in part by NASA grant 314401, US National Science Foundation grants EIA-0080123 and IIS-0100436, and by a grant from the Boeing Corporation. The authors thank the associate editor and the anonymous reviewers for their insightful comments.

\section{REFERENCES}

[1] C. Batini, S. Ceri, and S.B. Navathe, Conceptual Database Design: An Entity-Relationship Approach. Benjamin/Cummings Publishing Company, 1992.

[2] M.H. Böhlen, C.S. Jensen, and R.T. Snodgrass, "Temporal Statement Modifiers," ACM Trans. Database Systems, vol. 25, no. 4, pp. 407-456, 2000.

[3] P.P. Chen, "The Entity-Relationship Model-Toward a Unified View of Data," ACM Trans. Database Systems, vol. 1, no. 1, pp. 9-36, 1976.

[4] C. Claramunt, C. Parent, and M. Thériault, "An Entity-Relationship Model for Spatio-Temporal Processes," Data Mining and Reverse Eng.: Searching for Semantics, Proc. IFIP TC2/WG2.6 Seventh Conf. Database Semantics (DS-7), pp. 455-475, 1997.

[5] F.A. D'Agnese, C.C. Faunt, A.K. Turner, and M.C. Hill, "Hydrogeologic Evaluation and Numerical Simulation of the Death Valley Regional Ground-Water Flow System, Nevada and California," US Geological Survey Water Resources 96-4300, 1997.

[6] C.E. Dyreson, W.S. Evans, H. Lin, and R.T. Snodgrass, "Efficiently Supporting Temporal Granularities," IEEE Trans. Knowledge and Data Eng., vol. 12, no. 4, pp. 568-587, July/Aug. 2000.

[7] R. Elmasri and S.B. Navathe, Fundamentals of Database Systems. second ed. Redwood City, Calif.: Benjamin/ Cummings Publishing Co., 1994.

[8] S.K. Gadia, "A Homogeneous Relational Model and Query Languages for Temporal Databases," ACM Trans. Database Systems, vol. 13, no. 4, pp. 418-448, 1988.

[9] S.C. Guptill, "Multiple Representations of Geographic Entities through Space and Time," Proc. Fourth Int'l Symp. Spatial Data Handling, pp. 859-868, 1990.
[10] A.F. Hutchings and S.T. Knox, "Creating Products-Customers Demand," Comm. ACM, vol. 38, no. 5, pp. 72-80, 1995.

[11] C.S. Jensen, C.E. Dyreson, M. Bohlen, J. Clifford, R. Elmasri, S.K. Gadia, F. Grandi, P. Hayes, S. Jajodia, W. Kafer, N. Kline, N. Lorentzos, Y. Mitsopoulos, A. Montanari, D. Nonen, E. Peresi, B. Pernici, J.F. Roddick, N.L. Sarda, M.R. Scalas, A. Segev, R.T. Snodgrass, M.D. Soo, A. Tansel, R. Tiberio, and G. Wiederhold, "A Consensus Glossary of Temporal Database Concepts-February 1998 Version," Temporal Databases: Research and Practice, O. Etzion, S. Jajodia, and S. Sripada, eds., Springer-Verlag, 1998.

[12] J. Jing, A. Helal, and A.K. Elmagarmid, "Client-Server Computing in Mobile Environments," ACM Computing Surveys, vol. 31, no. 2, pp. 117-157, 1999.

[13] S. Juhn and J. Naumann, "The Effectiveness of Data Representation Characteristics on User Validation," Proc. Sixth Int'l Conf. Information Systems, pp. 212-226, 1985.

[14] V. Khatri, "Bridging the Spatio-Temporal Semantic Gap: A Theoretical Framework, Evaluation and A Prototype System," Doctoral Thesis: Management Information Systems Dept., Tucson, Ariz.: Univ. of Arizona, p. 320, 2002.

[15] V. Khatri, S. Ram, and R.T. Snodgrass, "ST-USM: Bridging the Semantic Gap with a Spatio-Temporal Conceptual Model," TimeCenter Technical Report TR-64, 2001.

[16] V. Khatri, S. Ram, and R.T. Snodgrass, "Supporting User-Defined Granularities and Indeterminacy in a Spatiotemporal Conceptual Model," Annals of Math. and Artificial Intelligence, vol. 36, nos. 1-2, pp. 195-232, 2002.

[17] B. Kuipers, "Modeling Spatial Knowledge," Cognitive Science, vol. 2, pp. 129-153, 1978.

[18] Y.C. Lee and M. Isdale, "The Need for a Spatial Data Model," Proc. Canadian Conf. in GIS-911991, year?

[19] D.M. Mark and A.U. Frank, "Experiential and Formal Models of Geographic Space," Environment and Planning, vol. 23, no. 1, pp. 324, 1996.

[20] J.L. Mennis, D.J. Peuquet, and L. Qian, "A Conceptual Framework for Incorporating Cognitive Principles into Geographical Database Representation," Int'l J. Geographic Information Science, vol. 14, no. 6, pp. 501-520, 2000.

[21] D.J. Peuquet, "It's About Time: A Conceptual Framework for the Representation of Temporal Dynamics in Geographic Information Systems," Annals of the Assoc. of Am. Geographers, vol. 83, no. 3, pp. 441-461, 1994.

[22] S. Ram, "Intelligent Database Design Using the Unifying Semantic Model," Information and Management, vol. 29, no. 4, pp. 191-206, 1995.

[23] C.V. Ramamoorthy, A. Prakash, W. Tsai, and Y. Usuda, "Software Engineering: Problems and Perspectives," Computer, vol. 17, no. 10, pp. 191-209, Oct. 1984.

[24] J.F. Roddick and R.T. Snodgrass, "Schema Versioning," The TSQL2 Temporal Query Language, R.T. Snodgrass, ed., pp. 427-449, Boston: Kluwer Academic Publishers, 1995.

[25] P. Serrano-Alvarado, C. Roncancio, and M.E. Adiba, "Analyzing Mobile Transaction Supports for DBMS," Proc. 12th Int'l Workshop Database and Expert Systems Applications (DEXA 2001), pp. 595-600, 2001.

[26] A. Sheth, "Data Semantics: What, Where and How?," Proc. Sixth IFIP Working Conf. Data Semantics (DS-6), pp. 601-610, 1995.

[27] A. Silbershatz, H. Korth, and S. Sudarshan, Database System Concepts. third ed., WCB/McGraw Hill, 1997.

[28] R.T. Snodgrass and I. Ahn, "Temporal Databases," Computer, vol. 19 , no. 9, pp. 35-42, Sept. 1986.

[29] N. Tryfona and C.S. Jensen, "Conceptual Data Modeling for Spatiotemporal Applications," Geoinformatica, vol. 3, no. 3, pp. 245$268,1999$.

[30] J.W. van Roessel, "Design of a Spatial Data Structure Using the Relational Normal Form," Int'l J. Geographical Information Systems, vol. 1, no. 1, pp. 33-50, 1987.

[31] Y. Wand, D.E. Monarchi, J. Parsons, and C.C. Woo, "Theoretical Foundations for Conceptual Modeling in Information Systems Development," Decision Support Systems, vol. 15, no. 4, pp. 285-304, 1995.

[32] Y. Wand, V.C. Storey, and R. Weber, "On Ontological Analysis of the Relationship Construct in Conceptual Modeling," ACM Trans. Database Systems, vol. 24, no. 4, pp. 494-528, 1999.

[33] M.F. Worboys, H.M. Hearshaw, and D.J. Maguire, “ObjectOriented Data Modeling for Spatial Databases," Int'l J. Geographical Information Systems, vol. 4, no. 4, pp. 369-383, 1990. 


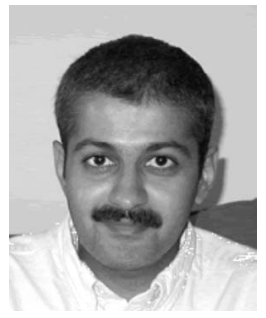

Vijay Khatri received the BE degree (electronics) from Malaviya Regional Engineering College, the management degree from the University of Bombay, and the PhD degree from the University of Arizona. $\mathrm{He}$ is an assistant professor of information systems in the Kelley School of Business at the Indiana University. Dr. Khatri has published articles in journals such as Information Systems, Annals of Mathematics and Artificial Intelligence, and Photogrammetric Engineering and Remote Sensing. His research centers on issues related to data semantics, semiotics and conceptual database design, temporal databases, and spatial databases. More specifically, his research involves developing techniques for management of data, especially for applications that need to organize data based on time and space. He serves on the editorial review board of the Journal of Data Management. $\mathrm{He}$ is a member of the IEEE and the IEEE Computer Society.

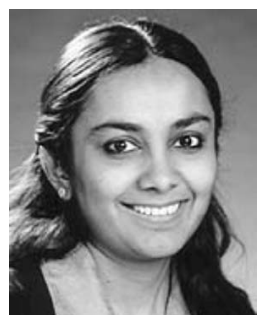

Sudha Ram received the BS degree in mathematics, physics, and chemistry from the University of Madras in 1979, PGDM from the Indian Institute of Management, Calcutta in 1981, and the PhD degree from the University of Illinois at Urbana-Champaign in 1985. She is the Eller Professor of Management Information Systems in the College of Business and Public Administration at the University of Arizona. Dr. Ram has published articles in such journals as Communications of the ACM, ACM Transactions on Information Systems, IEEE Transactions on Knowledge and Data Engineering, Information Systems Research (ISR), Information Systems, MIS Quarterly, and Management Science. Dr. Ram's research deals with modeling and analysis of databases for manufacturing, scientific, and business domains. Her research has been funded by IBM, NCR, US ARMY, NIST, US National Science Foundation, NASA, NIH, and $\mathrm{ORD}(\mathrm{CIA})$. Specifically, her research deals with interoperability among heterogeneous database systems, semantic modeling, data allocation, schema and view integration, intelligent agents for data management, and tools for database design. Dr. Ram serves on various editorial boards including Information Systems Research, Journal of Database Management, Information Systems Frontiers, and the Journal of Systems and Software. She is also the director of the Advanced Database Research Group at the University of Arizona. She is a member of the IEEE and the IEEE Computer Society.

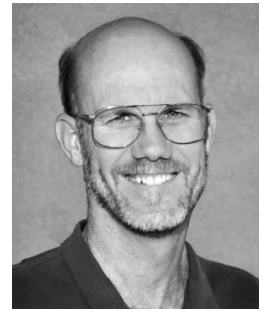

Richard T. Snodgrass received the BA degree in physics from Carleton College and the MS and $\mathrm{PhD}$ degrees in computer science from Carnegie Mellon University. He joined the University of Arizona in 1989, where he is a professor of computer science. He is an ACM fellow. Dr. Snodgrass is editor-in-chief of the ACM Transactions on Database Systems. He chairs the ACM SIGMOD Advisory Board and has served on the editorial boards of the International Journal on Very Large Databases and the IEEE Transactions on Knowledge and Data Engineering. He chaired the Americas program committee for the 2001 International Conference on Very Large Databases, chaired the program committees for the 1994 ACM SIGMOD Conference and the 1993 International Workshop on an Infrastructure for Temporal Databases, and was a vice-chair of the program committees for the 1993 and 1994 International Conferences on Data Engineering. He was ACM SIGMOD Chair from 1997 to 2001 and has previously chaired the ACM Publications Board and the ACM SIG Governing Board Portal Committee. He received the ACM SIGMOD Contributions Award in 2002. He chaired the TSQL2 Language Design Committee, edited the book, The TSQL2 Temporal Query Language (Kluwer Academic Press), and has worked with the ISO SQL3 committee to add temporal support to that language. He authored Developing Time-Oriented Database Applications in SQL (Morgan Kaufmann), was a coauthor of Advanced Database Systems (Morgan Kaufmann), and was a coeditor of Temporal Databases: Theory, Design, and Implementation (Benjamin/Cummings). He codirects TimeCenter, an international center for the support of temporal database applications on traditional and emerging DBMS technologies. His research interests include temporal databases, query language design, query optimization and evaluation, storage structures, and database design. He is a senior member of the IEEE and the IEEE Computer Society.

$\triangle$ For more information on this or any other computing topic, please visit our Digital Library at www.computer.org/publications/dlib. 